\title{
RESEARCH HIGHLIGHT Ventral pallidum: a promising target for addiction intervention
}

\author{
Dillon J. McGovern ${ }^{1}$ and David H. Root (D) \\ Neuropsychopharmacology (2019) 44:2151-2152; https://doi.org/10.1038/s41386-019-0528-z
}

The ventral pallidum (VP) is part of a "final common pathway" of relapse behavior [1]. The extinction-reinstatement paradigm provides a reliable method to study the neurobiology of drug relapse [2]. Recently, variations on the extinction component have been devised to more closely approximate the experiences of human users. The voluntary abstinence model probabilistically pairs an aversive outcome (footshock) concurrently with drug reward [3]. In this model, all rodents eventually refrain from drug selfadministration. However, subsets of subjects can be classified as relatively punishment resistant or sensitive based on the maximum level of shock current tolerated before they choose to abstain. Rather than experimenter-imposed extinction, punishment offers the opportunity to examine reinvigorated drug seeking following voluntary abstinence. Further, there is evidence to suggest that drug seeking after punishment induced-voluntary abstinence recruits different neuronal circuits than those recruited after experimenter-imposed extinction [3]. Here, Farrell and colleagues [4] investigated the role of the VP in cue, context, and drug primed reinstatement after punishment-induced abstinence.

Before discussing the results, it is important to appreciate the difficulty of targeting and identifying the VP. There are no clear VP boundaries within Nissl-stained tissue. In addition, several neurochemically-distinct VP subdivisions exist with individual circuitries and differential densities of VP cell-types [5]. Farrell et al. used substance $P$ immunoreactivity to identify the entire VP, which was used to localize viral designer receptor (DREADD) injections within or outside of VP. In other studies, they combined substance $P$ immunoreactivity with neurotensin and c-Fos immunoreactivity to aid in identifying relapse-activated cells within neurochemically-distinct subdivisions of the VP. The authors also performed in situ hybridization to identify the GABAergic and glutamatergic identities of DREADD-labeled neurons. Most DREADD-labeled neurons were GABAergic but a small subset of glutamatergic neurons was also labeled.

The authors activated the hM4D-Gi DREADD to inhibit VP neurons during different types of relapse-provoking stimuli following voluntary abstinence in male and female rats. Critically, they found that when DREADD expression was localized to substance $P$ immunolabeled VP neurons, that relapse was reduced. However, when the DREADD was localized largely outside of the substance $P$ immunolabeled VP, relapse was unaffected. Thus, without substance $P$ immunolabeling it would be nearly impossible to identify whether VP or surrounding basal forebrain structures are critical for relapse following voluntary abstinence. The identification of most DREADD-expressing neurons as GABAergic suggests that their activation is required for relapse after voluntary abstinence.

In addition, VP inhibition decreased abortive lever pressing, an approach-avoidance behavior involving approaching and reaching but retracting from a drug-paired lever. We have shown that subsets of VP neurons decrease or increase firing specifically during approach or retreat behavior of a drug-paired device [6]. Given that VP GABA and glutamate neurons can facilitate or restrain reward motivation [7], the reduction in concurrent approach and avoidance behaviors during abortive lever pressing might reflect altering the neuronal activity of both VP GABAergic and glutamatergic cell-types, as accomplished here.

To investigate the roles of VP subdivisions in relapse, c-Fos expression within the neurochemically-distinct VP subdivisions after different types of relapse (provoked by context, cue, and cocaine priming) was analyzed. Dorsolateral VP and ventrolateral VP showed elevated c-Fos immunoreactivity during all relapse tests compared to home cage controls. We have found that dorsolateral VP neurons are highly sensitive to drug-seeking and drug-taking behaviors, though typically decrease firing during these behaviors [6]. Nucleus accumbens core neurons are largely excited by drug-seeking responses and their downstream target of dorsolateral VP neurons are mostly inhibited by drug-seeking responses $[5,6]$. Further, inhibiting the GABAergic core pathway to dorsolateral VP blocks a cocaine and conditioned cue combination reinstatement [8]. The discrepancy between decreased firing specifically during approaching and responding to a drug-taking device and elevated c-Fos shown here, following reinstatement, may be due to either differences in timescale (milliseconds to hours) or measurement (firing versus c-Fos immunoreactivity).

Dividing the VP rostrally and caudally revealed elevated c-Fos immunoreactivity in the rostral over the caudal VP selectively during relapse within the punishment context without drugassociated cues (also found for the ventromedial VP). Prior work from this group had shown that neurons in rostral VP $\rightarrow$ VTA pathway show elevated c-Fos during cue-induced reinstatement over neurons in the caudal VP $\rightarrow$ VTA projection [9]. These results suggest that specific heterogeneous populations of VP neurons mediate distinct aspects drug-seeking behavior.

Additional questions remain. For instance, the roles of distinct VP cell-types from discrete VP subdivisions and their upstream or downstream pathways involved in relapse following voluntary abstinence. As discussed by the authors, the circuit mechanisms by which VP inhibition reduces relapse remains puzzling. Activation of the GABAergic nucleus accumbens core projection to dorsolateral VP is necessary for reinstatement after extinction [8]. Yet, accumbal inhibition of VP neurons would mimic the effects of the inhibitory DREADD in VP that blocked relapse after voluntary abstinence shown here. Further interrogation of accumbens/VP interactions, including the involvement of coreleased peptides and other afferents will be necessary to understand how the nucleus accumbens, VP, and other involved structures together mediate drug-seeking behavior.

\footnotetext{
${ }^{1}$ Department of Psychology and Neuroscience, University of Colorado, 2860 Wilderness PI, Boulder, CO 80301, USA
}

Correspondence: David H. Root (David.Root@Colorado.edu)

Received: 11 September 2019 Accepted: 12 September 2019

Published online: 26 September 2019 
As part of the final common pathway of drug-seeking behavior, VP manipulations may represent a promising direction for developing therapeutic targets. As such, Farrell et al. [4] demonstrate that the most punishment-resistant rats showed the largest decreases in drug seeking by VP inhibition. While females comprised a larger majority of punishment resistant rats, there were no overall sex differences observed between the effects of VP inhibition. Nonetheless, the results are consistent with a role of VP in drug-seeking/relapse behavior and suggest that VP intervention may be efficacious in users that continue to seek drugs despite negative consequences.

\section{FUNDING AND DISCLOSURE}

This Research Highlight was supported by the Webb-Waring Biomedical Research Award from the Boettcher Foundation, the CO-PILOT award from the Colorado Clinical and Translational Sciences Institute, and a 2020 NARSAD Young Investigator grant from the Brain and Behavior Research Foundation. The authors have no financial interests to be disclosed.

\section{ADDITIONAL INFORMATION}

Publisher's note Springer Nature remains neutral with regard to jurisdictional claims in published maps and institutional affiliations.

\section{REFERENCES}

1. Kalivas K, Volkow PW, D N. The neural basis of addiction: a pathology of motivation and choice. Am J Psychiatry. 2005;162:1403-13.

2. Shaham Y, Shalev U, Lu L, de Wit H, Stewart J. The reinstatement model of drug relapse: history, methodology and major findings. Psychopharmacology. 2003;168:3-20. https://doi.org/10.1007/s00213-002-1224-x

3. Marchant NJ, Campbell EJ, Pelloux Y, Bossert JM, Shaham Y. Context-induced relapse after extinction versus punishment: similarities and differences. Psychopharmacology. 2019;236:439-48.

4. Farrell MR, et al. Ventral pallidum is essential for cocaine relapse after voluntary abstinence in rats. Neuropsychopharmacology 1-13 (2019).

5. Root DH, Melendez Rl, Zaborszky L, Napier TC. The ventral pallidum: subregionspecific functional anatomy and roles in motivated behaviors. Prog Neurobiol. 2015;130:29-70. https://doi.org/10.1016/j.pneurobio.2015.03.005

6. Root $\mathrm{DH}$, et al. Differential roles of ventral pallidum subregions during cocaine selfadministration behaviors. J Comp Neurol. 2013;521:558-88. https://doi.org/ 10.1002/cne.23191

7. Faget $\mathrm{L}$, et al. Opponent control of behavioral reinforcement by inhibitory and excitatory projections from the ventral pallidum. Nat Commun. 2018;9:849.

8. Stefanik MT, Kupchik YM, Brown RM, Kalivas PW. Optogenetic evidence that pallidal projections, not nigral projections, from the nucleus accumbens core are necessary for reinstating cocaine seeking. J Neurosci. 2013;33: 13654-62.

9. Mahler SV, et al. Designer receptors show role for ventral pallidum input to ventral tegmental area in cocaine seeking. Nat Neurosci. 2014;17:577-85. https://doi.org/ 10.1038/nn.3664 